\title{
THE RELATIONSHIP BETWEEN THE LOCATION OF OBSTACLE AND INDOOR PARTICLE DEPOSITION
}

\author{
Je-Ee Ho \\ Department of Mechanical Engineering, National Ilan University, Ilan, Taiwan, R.O.C., jeho@niu.edu.tw \\ Hong T. Young \\ Department of Mechanical Engineering, National Taiwan University, Taipei, Taiwan, R.O.C.
}

Follow this and additional works at: https://jmstt.ntou.edu.tw/journal

Part of the Mechanical Engineering Commons

\section{Recommended Citation}

Ho, Je-Ee and Young, Hong T. (2007) "THE RELATIONSHIP BETWEEN THE LOCATION OF OBSTACLE AND INDOOR PARTICLE DEPOSITION," Journal of Marine Science and Technology. Vol. 15: Iss. 1, Article 4.

DOI: $10.51400 / 2709-6998.2029$

Available at: https://jmstt.ntou.edu.tw/journal/vol15/iss1/4

This Research Article is brought to you for free and open access by Journal of Marine Science and Technology. It has been accepted for inclusion in Journal of Marine Science and Technology by an authorized editor of Journal of Marine Science and Technology. 


\title{
THE RELATIONSHIP BETWEEN THE LOCATION OF OBSTACLE AND INDOOR PARTICLE DEPOSITION
}

\author{
Je-Ee Ho* and Hong T. Young**
}

Key words: isolated zone, sucking zone, minor closure region, vortex region.

\section{ABSTRACT}

An appropriate ventilation system design not only increases the indoor ventilation effect but effectively improves the particle concentration distribution. The locations of indoor furniture are used to analyze the indoor airflow and particle distribution by numerical skill in this study. Also, a $45 \mathrm{~cm} \times 45 \mathrm{~cm} \times 45 \mathrm{~cm}$ chamber is setup as experiment apparatus which is made by acrylic resin board with 16 holes connected to supply air flow. In order to simulate the furniture effect, some wooden blocks are located at the chamber. The temperature and humidity sensors are connected to the data acquisition system with a personal computer to record all the test data. The experimental results show that much more deposited mass will be observed in isolated zone than that in other area and the maximum simulated suspending particles will exist at the vortex zone where the aerosol distribution keeps almost constant.

\section{INTRODUCTION}

The particle size and concentration are very important factors for human living in an air-tight environment. It not only influences the human health but also does in some precise manufacturing process. Therefore, the research on the particle behavior for considering the convective effect is very necessary in some specific environment such as health facilities.

At 1988, Dunn [3] reported the influence of moisture effect on particle deposition rate. The moisture decreased particle deposition rate because it changed heat convection effect in the air. Axley [1] examined particle concentration with several important parameters such as indoor airflow, temperature and humidity. By his experiment showed that the strength of airflow would be most significant influence on the aerosol

Paper Submitted 03/14/06, Accepted 06/12/06. Author for Correspondence: Je-EeHo.E-mail: jeho@niu.edu.tw.

*Department of Mechanical Engineering, National Ilan University, Ilan, Taiwan, R.O.C.

**Department of Mechanical Engineering, National Taiwan University, Taipei, Taiwan, R.O.C. distribution indoors. Kurabuchi et at. [4] reported that the particle diffusion increased as the temperature increased and decreased the particle deposition rate. At 2001, Lin [5] performed numerical simulation for analyzing the indoor air flow field; some important parameters including the inlet, outlet location, air jet speed, temperature and the efficiency of the pollutants removal were investigated. This paper primarily studies the effect of the locations of obstacles on the particle deposition in an enclosure space where the temperature and humidity are treated as minor factors. The airflow pattern and particle concentration in the isolated zone are also predicted by numerical simulation. Meantime, the experimental data from scaled model are used to compare the predicted results with different locations of obstacles indoors. The final conclusions may be as important references for indoor building design.

\section{NUMERICAL MODELS}

The numerical model includes mass conservation equation, momentum equation, energy equation and concentration equation. Total of seven equations were used to solve the flow field and concentration distribution with $\kappa-\varepsilon$ turbulent model [2]. Through the dimensionless analysis, the above seven equations were integrated into Re number, Pr number and Sc Schmidt number.

\section{Dimensionless governing equation}

Continuity equation:

$$
\frac{\partial \hat{u} i}{\partial \hat{x} j}=0
$$

Momentum equation:

$$
\frac{\partial \hat{u} i}{\partial t}+\frac{\partial \hat{u} i \partial \hat{u} j}{\partial \hat{x} j}=\frac{-\partial P}{\partial \hat{x} i}+\frac{\partial}{\partial \hat{x} j}\left\{\hat{v}_{e f f}\left(\frac{\partial \hat{u} i}{\partial \hat{x} j}+\frac{\partial \hat{u} j}{\partial \hat{x} i}\right)\right\}
$$

$\kappa$ equation:

$$
\frac{\partial k \hat{u} j}{\partial \hat{x} j}=\frac{\partial}{\partial \hat{x} j}\left[P_{k} \frac{\partial \hat{k}}{\partial \hat{x} j}\right]+\hat{v}_{t} S-\hat{\varepsilon}
$$


$\varepsilon$ equation:

$\frac{\partial \varepsilon \hat{u} j}{\partial \hat{x} j}=\frac{\partial}{\partial \hat{x} \hat{j}}\left[P_{\varepsilon} \frac{\partial \hat{\varepsilon}}{\partial \hat{x} j}\right]+\frac{\hat{\varepsilon}}{\hat{\kappa}}\left(c_{1} \hat{v}_{t}-c_{2} \widehat{\varepsilon}\right)$

Concentration Eq.:

$$
\frac{\partial c \hat{u} j}{\partial \hat{x} j}=\frac{\partial}{\partial \hat{x} j}\left[\alpha_{e f f} \frac{\partial c}{\partial \hat{x} j}\right]+\hat{v}_{g}-\frac{\partial c}{\partial \hat{z}}
$$

\section{Numerical process}

The SIMPLE algorithm developed by Partankar [6] was applied to solve the equations. In order to avoid the unreasonable zigzag or wavy velocity during the convergent process, the staggered grid method was used in this study. The computational process kept going until the relative error less than $1 / 1000$ was reached in all the physical terms. Although the calculated time was much longer than usual methods, the result seemed to be better and more precise in physical quantity solutions.

\section{SCALED EXPERIMENTAL MODEL}

The scaled experimental model was formed by six $45 \mathrm{~cm} \times 45 \mathrm{~cm}$ acrylic resin boards with 16 air supply

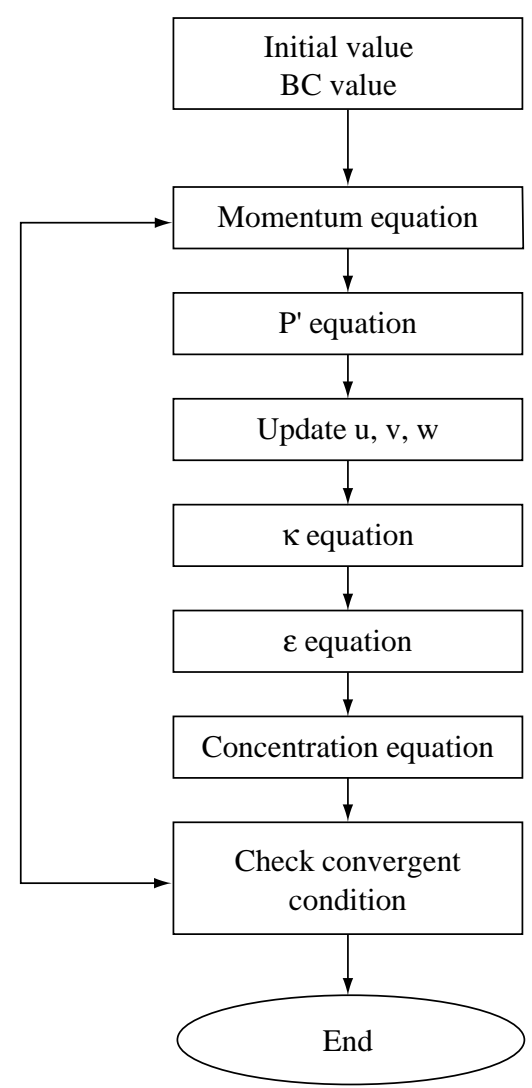

Fig. 1. Computational flow chart. holes on each side. An air compressor was used as the air supply source with a mixing box controller. The hot wire anemometers were equipped on the air supply outlet to measure the air velocities. The standard particles, F400, were used as the experimental particle sample. Before being injected into the chamber, the particles would be needed to be mixed with air in the mixing box. Meantime, the capture cups set among the wooden obstacles collected the dropped particles to quantify the depositing concentration every 30 minutes. The detailed experimental setup is shown in Figure 2.

\section{RESULTS AND DISCUSSION}

\section{Simulation results}

After airflow is driven from inlet, the jet will form a strong vortex at the center location of the enclosure as shown in Figure 3. When the inlet jet moves to wall, the flow will turn to the downward direction and then a clockwise circulation will be observed in the space. As for the aerosol distribution in Figure 4, the inlet flow with aerosol concentration $200 \mu \mathrm{g} / \mathrm{m}^{3}$ travels along the flow path (D to A) and it increases to $425 \mu \mathrm{g} / \mathrm{m}^{3}$ on the opposite wall by the stagnation effect. During flow path, the concentration contour presents much finer in the direction normal to inlet flow path. It implies that

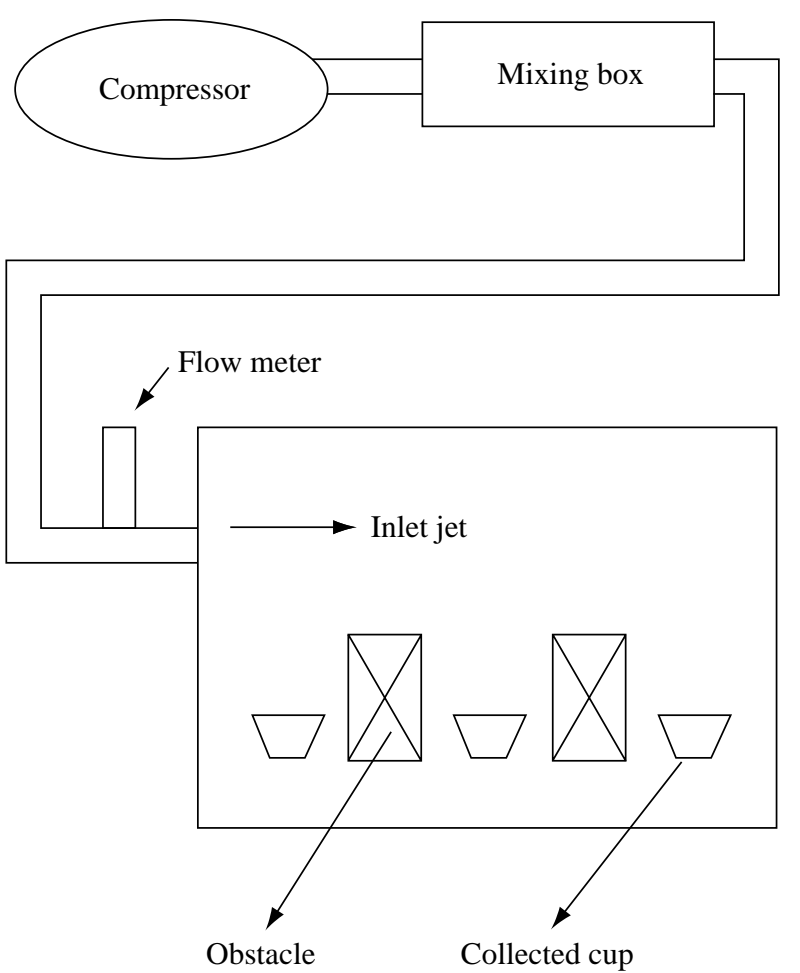

Fig. 2. Scaled model chamber. 


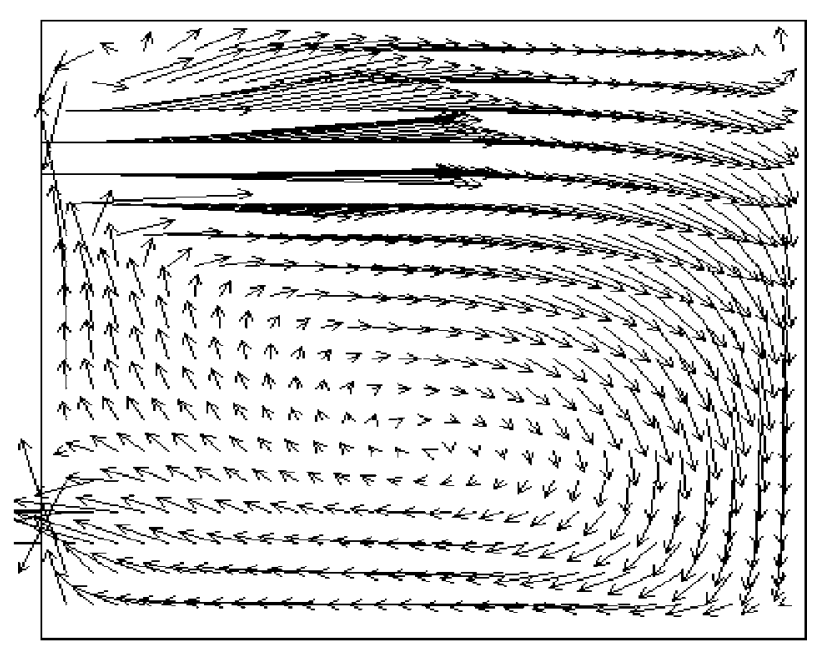

Fig. 3. Simulated flow field without obstacles for $U_{\text {inlet }}=0.3 \mathrm{~m} / \mathrm{s}$.

the convective effect presents much larger than the diffusion effect does, but the diffusion terms become dominated in normal to the inlet flow direction. Along the path $\mathrm{A} \rightarrow \mathrm{B}$ which is the same as gravitational direction, most of aerosol enhanced by the inlet flow will deposit at there and the particle concentration obviously increases to $600 \mu \mathrm{g} / \mathrm{m}^{3}$. This area we treat it as a depositing zone. The other side to $\mathrm{AB}$ path is the sucking zone where is under the inlet and the airflow is circulated upwards (from $\mathrm{C}$ to $\mathrm{D}$ ) there. Due to against the gravitational direction, the more particle concentration (about $500 \mu \mathrm{g} / \mathrm{m}^{3}$ along C to D) is suspended in the air and less particles will be deposited on the floor. As for vortex center, the particles fluctuate around this area where makes the lower particle depositing rate and higher particle concentration suspended in the air. As shown in Figure 4, the highest particle concentration $650 \mu \mathrm{g} / \mathrm{m}^{3}$ uniformly distributes at the vortex center.

From above discussion, the modeling space without obstacles will be summarized as three important regions, they are depositing zone, sucking zone and vortex zone.

When the obstacles are placed in the space, there are several characteristic enclosures should be worth mentioned. The first is major isolated zone, the second is minor closure region and the third is sucking zone. As Figures 5 and 6 released, the major isolated region is located between the obstacle and the wall where is normal to the flow direction. In this, a strong vortex with both different behaviors will be observed. One is to deposit most aerosol particles to the floor which makes the maximum particle deposition; the other is to circulate the deposited mass up to the air which results in more suspending particle concentration about 400

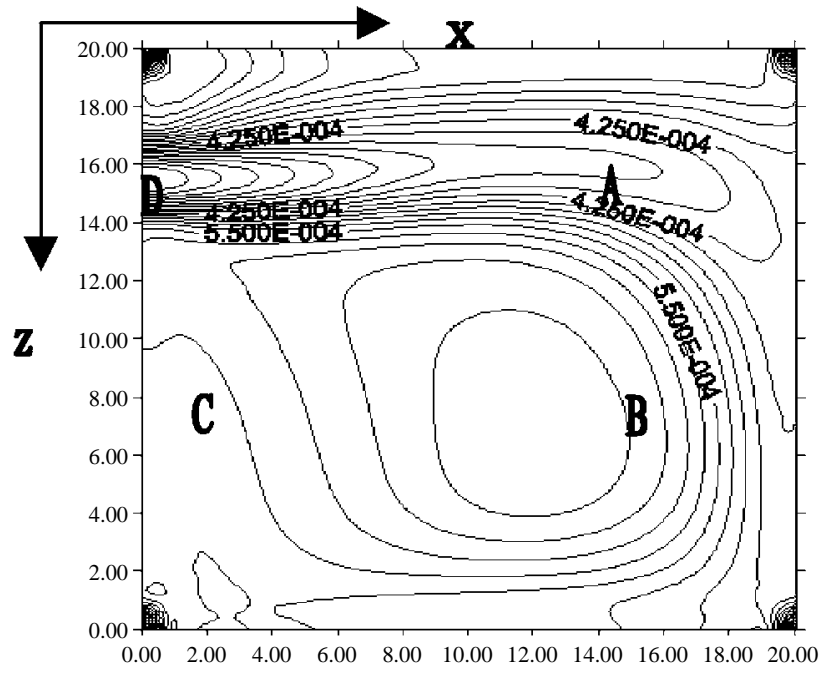

Fig. 4. Simulated aerosol distribution without obstacles for $\mathrm{U}_{\text {inlet }}=0.3 \mathrm{~m} / \mathrm{s}$.

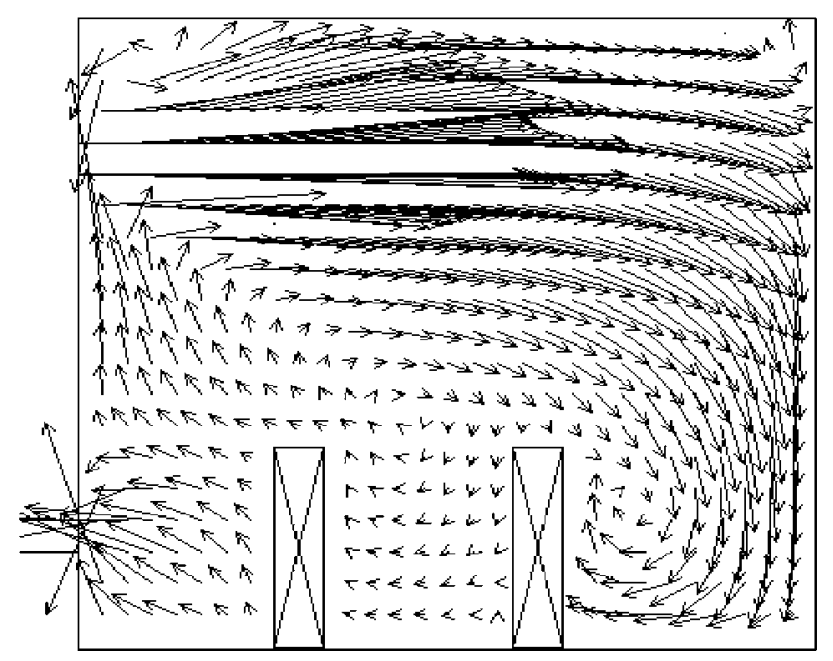

Fig. 5. Air flow field with obstacles for $U_{\text {inlet }}=0.3 \mathrm{~m} / \mathrm{s}$.

$\mu g / m^{3}$ in. The minor closure region appears between the blocks where the weaker convection is induced and the diffusion effect is only transportation mechanism that causes the lower deposition and less suspended particle concentration $300 \mu \mathrm{g} / \mathrm{m}^{3}$. The sucking zone is located beneath and close to the inlet where aerosol particles will be sucked upwards and suspended in the air, the maximum suspending concentration usually keeps about $500 \mu \mathrm{g} / \mathrm{m}^{3}$ in this region.

\section{Experimental results}

As above simulating description, several areas presenting significant difference with aerosol distribution have been discussed. In experimental model, we 


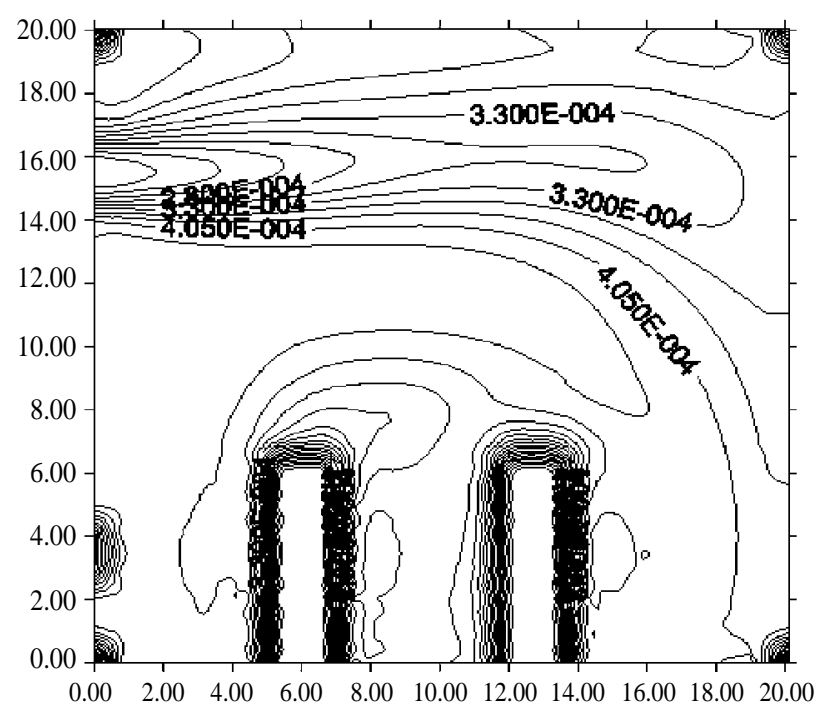

Fig. 6. Aerosol distribution with obstacles for $U_{\text {inlet }}=0.3 \mathrm{~m} / \mathrm{s}$.

set up the measuring system to collect the deposited mass at those corresponding regions to check the simulating results. If the test chamber without any obstacles inside is considered as shown in Figure 7, the depositing region appears at location 2 and 3 with more deposited mass $0.53 \mathrm{~g}, 0.58 \mathrm{~g}$ collected respectively. On the other, the less deposited mass $0.38 \mathrm{~g}, 0.39 \mathrm{~g}$ are obtained at location 1 and 5 where are close to the sucking regions. Because not any isolated region has been formed inside the modeling space, the particles deposited seem to be not significant difference everywhere except a little more in depositing area.

The obstacles are placed with the same side and normal to the inlet jet as shown in Figure 8. Beside the flow strength will be decreased by the obstacles, the isolated zones (location 3) and sucking zones (location $1,5)$ will be formed in the chamber. The major isolated zone has the highest particle deposition $0.81 \mathrm{~g}$, and $0.39 \mathrm{~g}$ collected at minor closure zone (location 2 ). As to the sucking effect, the lower particles $0.28 \mathrm{~g}, 0.29 \mathrm{~g}$ was obtained respectively at location 1,5 . Due to those characteristic zones appear in the space; their corresponding mass collected will behave significant difference.

When setting the obstacles with other side to inlet jet as indicated in Figure 9, we find that all the characteristic zones mentioned above will be appear in the space. The major isolated region has been transformed to location 3 whose deposited mass is lowed to $0.51 \mathrm{~g}$, This is because part of aerosol particles enhanced in the inlet jet about $0.4 \mathrm{~g}$ had been dropped down at the deposit zone(location 2) in advance. As for the minor closure zone (location 4), it is little affected by the

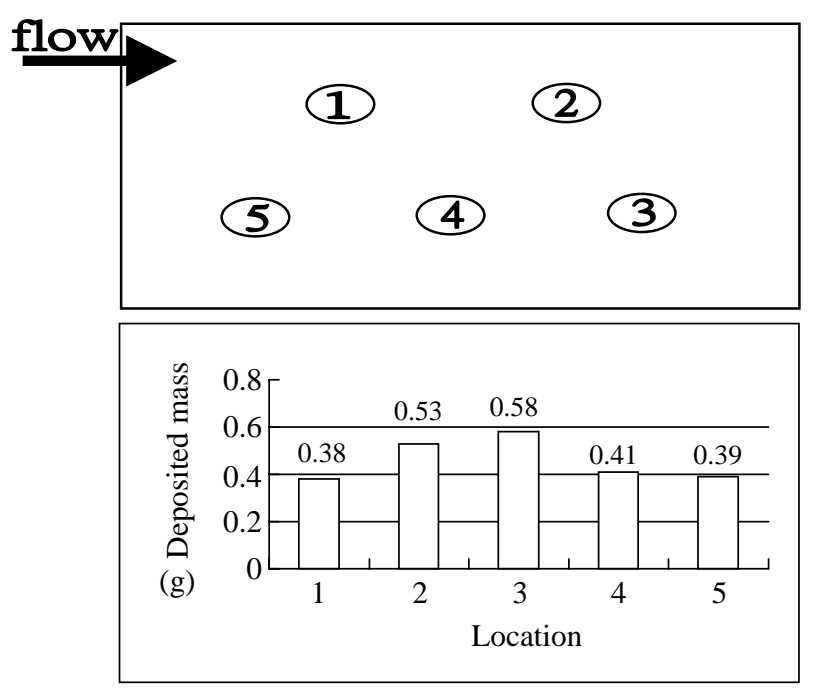

Fig. 7. The collected deposited mass without any obstacles at indoor space.

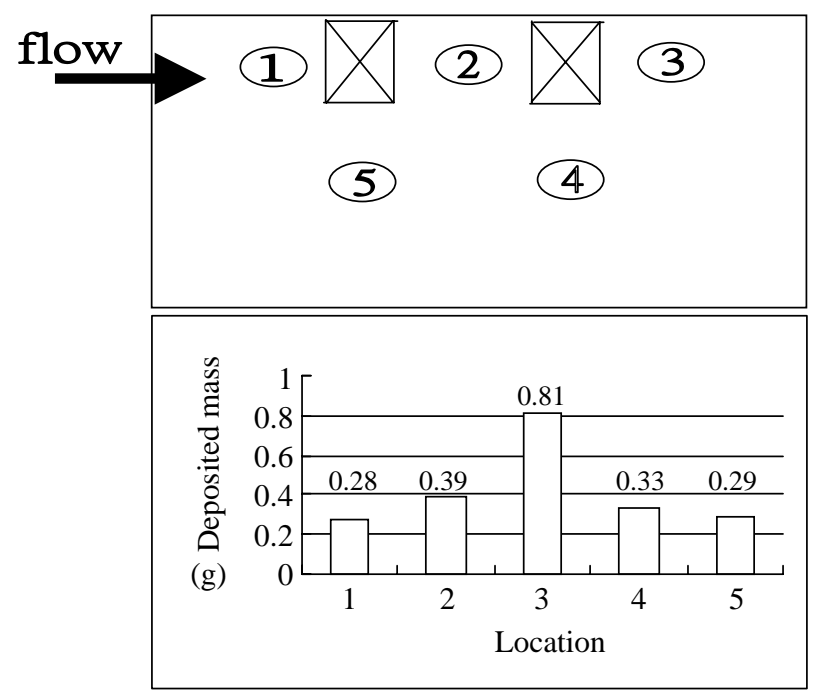

Fig. 8. The collected deposited mass versus with obstacles which is the same side and normal to the inlet flow.

recirculation flow and only $0.36 \mathrm{~g}$ aerosol particles are diffused to the floor. The lowest deposited mass is still observed at the sucking zone (location 5) where the sucking effect works and its value almost keeps $0.3 \mathrm{~g}$ there. By comparing with the arrangement in Fig8, we notice that the depositing distribution is more uniform in Figure 9 because half of the aerosols in the inlet flow will be mean distributed at location 2 and location 3 instead of being concentrated at location 3 in Figure 8 .

Replaced the obstacles with parallel to inlet flow as in Figure 10, the area near location 2 forms a large 


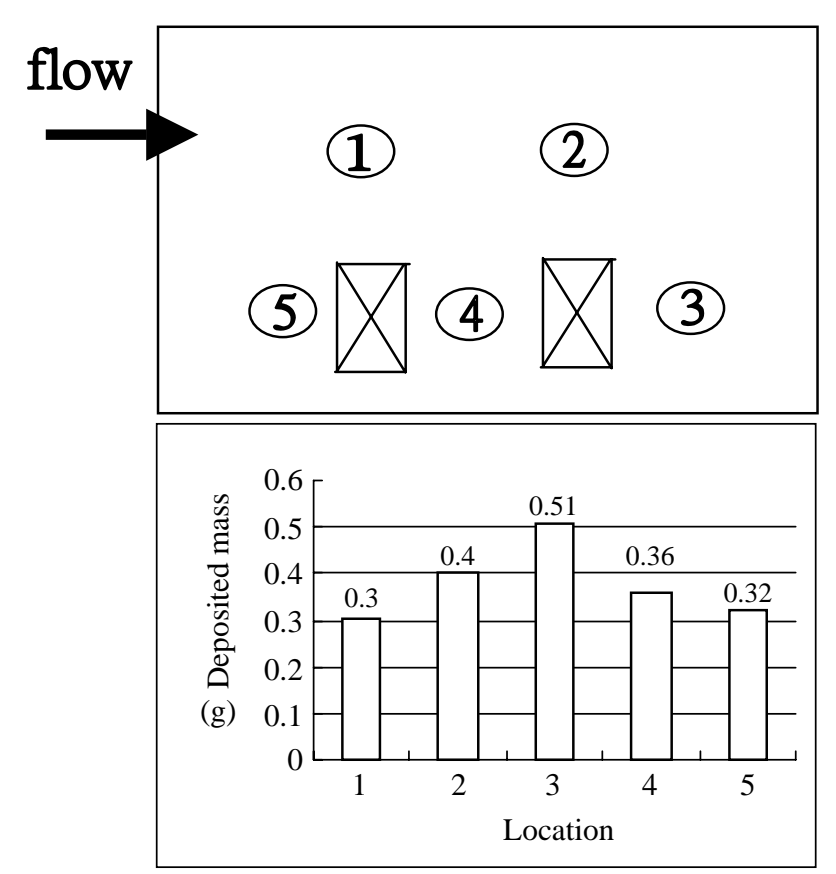

Fig. 9. The collected deposited mass versus with which is in other side and normal to the inlet.

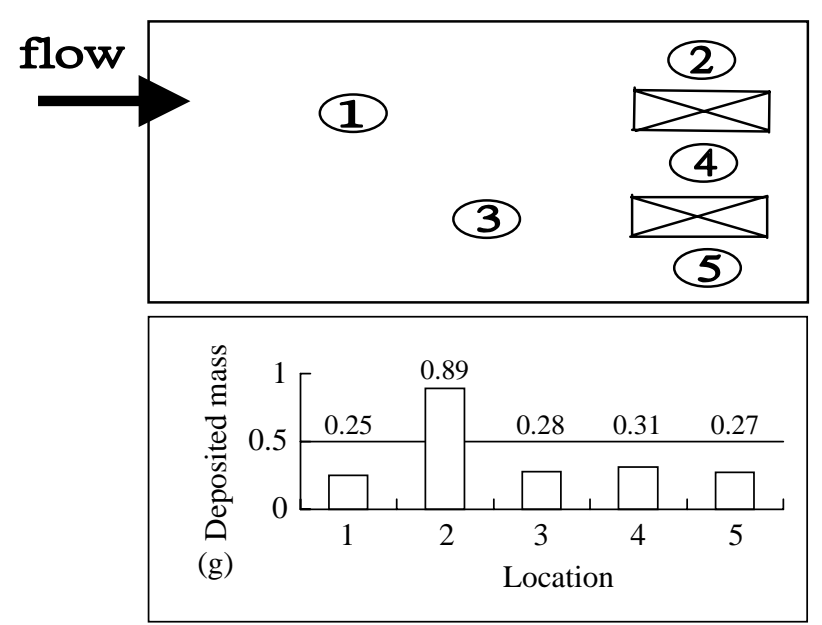

Fig. 10. The collected deposited mass versus with obstacles which is in opposite side and parallel to the inlet flow.

closure zone where the isolated zone appears and most particles in the jet about $0.89 \mathrm{~g}$ will be gathered on this area. The strength of airflow will be decreased by the stagnation effect at location 2 and causes weaker vortexes in zones 4 and 5. It reduces the amount of particle deposition in those areas with $0.31 \mathrm{~g}$ in zone 4 and 0.27 $\mathrm{g}$ in zone 5. The lowest particle deposition only $0.25 \mathrm{~g}$ is observed at zone 1 where is close to the sucking region.

In Figure 11, four obstacles are placed inside

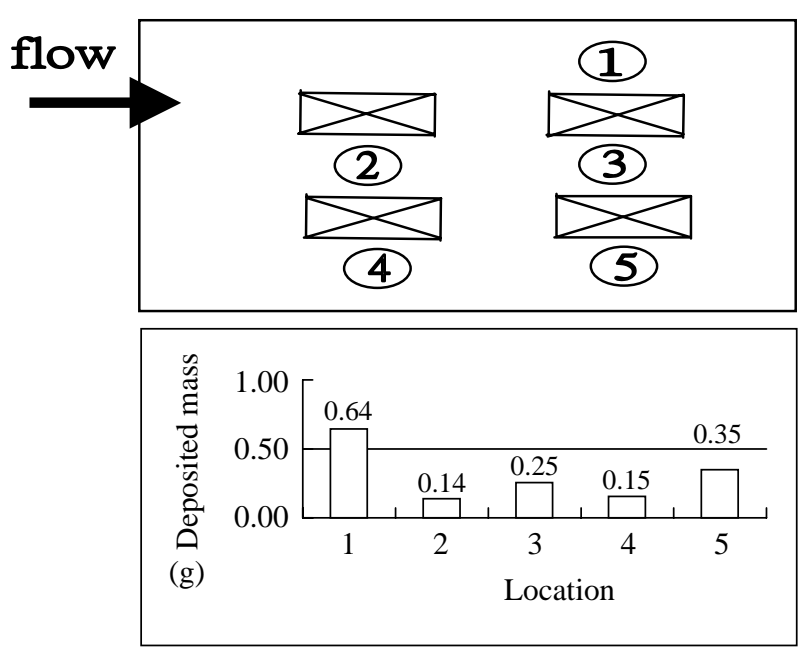

Fig. 11. The collected deposited mass versus with obstacles uniformly distributed at indoor space and parallel to the inlet flow.

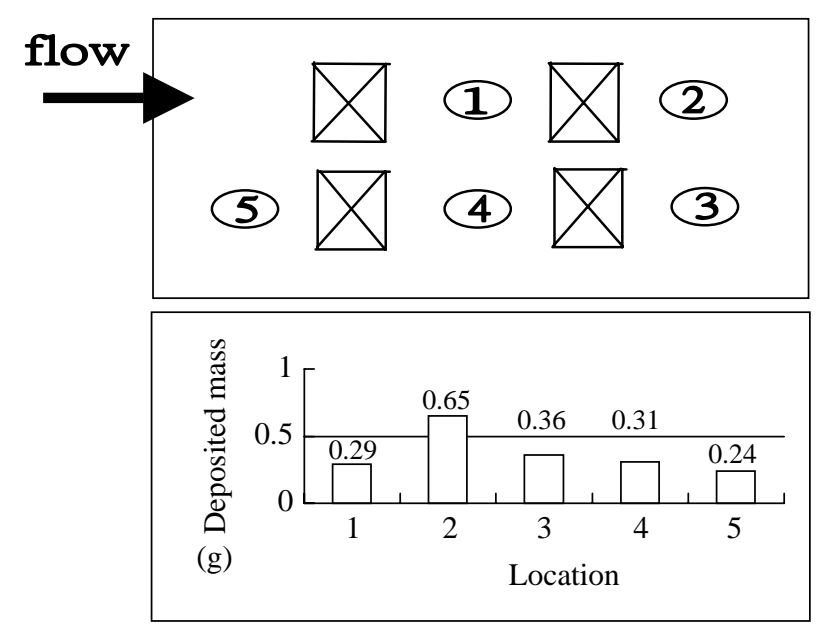

Fig. 12. The collected deposited mass versus with obstacles uniformly distributed at indoor space and normal to the inlet flow.

chamber evenly and parallel to the air jet direction. Comparison to Figure 10, a non-complete major isolated zone with some distance from the wall is found near zone 1 where the highest particle deposition $0.64 \mathrm{~g}$ presents. Another non-complete closure region with lower strength of air flow is observed at zone 5 , it contains $0.35 \mathrm{~g}$ particle deposition. Close to the sucking area, zones 2 and 4 have least deposited mass only about 0.15 g respectively.

When the obstacles are arranged vertically to air flow direction as Figure 12, the major isolated region zone 2 where the inlet jet is strongly impinged by the wall makes the most aerosol particles drop down, and deposit maximum particles about $0.65 \mathrm{~g}$. Another isolated region is formed at zone 3 with the air flow 
stagnated here again and also deposits more particles about $0.36 \mathrm{~g}$ which is less than that in zone 2 . As the flow travels from zone 3 to zone 5 where locates closer to the sucking area, the collected mass will become less. Compared with the Figure 11, the more deposited mass will be collected as arrangement in Figure 12. That is due to the obstacles normal to the flow direction reduce the circulation and cause the more aerosol particles to drop down.

\section{CONCLUSIONS}

1. The amount of particle deposition is strongly dependent on the direction of airflow. When the airflow direction headed to floor, it will increase the amount of particle deposition and the gradient of concentration in air will varies significantly.

2. When the airflow is close to the sucking area, the deposited mass will become less and the concentration of suspended particle will be higher and almost remain constant.

3. In the center of flow vortex, the unstable circulated airflow causes less particle deposition and increases particle concentration in air.

4. When no obstacle is inside the chamber, the particle deposition seems to approach a mean distribution on the floor. Otherwise, if the obstacles are arranged inside the room, the drop distribution significantly varies in different region, especially around isolated zone.

5. When the obstacles are placed parallel to inlet jet and close to the wall, the major isolated zones are formed. It results in more deposited mass and suspended particles in this area.

6. If obstacles are arranged vertically to air flow, the circulating flow will be retarded by the obstacles and its strength will be rapidly decreased along the flow path. That results in more aerosols deposited.

\section{NOMENCLATURE}

$\mathrm{xj}$ : coordinate in $\mathrm{j}$ component $(\mathrm{m})$

uj: local velocity in $\mathrm{j}$ component $(\mathrm{m} / \mathrm{s})$

P: local air pressure $\left(\rho_{a}\right)$

$\kappa$ : turbulence energy $(\mathrm{m} / \mathrm{s})^{2}$

$\varepsilon$ : dissipation rate of turbulence energy $\left(\mathrm{m} / \mathrm{s}^{2}\right)$

c: concentration for passive contaminant $\left(\mathrm{g} / \mathrm{m}^{3}\right)$

$v$ : instantaneous viscosity of air $\left(\mathrm{m}^{2} / \mathrm{s}\right)$

D: diffusion coefficient for passive contaminant $\left(\mathrm{m}^{2} / \mathrm{s}\right)$

$\alpha$ : thermal diffusivity of air $\left(\mathrm{m}^{2} / \mathrm{s}\right)$

$\mathrm{d}$ : diameter of contaminant particle $\left(\mathrm{m}^{2} / \mathrm{s}\right)$

$\rho \mathrm{p}$ : density of contaminant particle $\left(\mathrm{kg} / \mathrm{m}^{3}\right)$

$\mu$ : dynamic viscosity of air $(\mathrm{kg} / \mathrm{ms})$

$\mathrm{L}_{\mathrm{o}}$ is the width of the inlet opening $(\mathrm{m})$
$\mathrm{U}_{\mathrm{o}}$ is the inlet air velocity $(\mathrm{m} / \mathrm{s})$

$\rho_{o}$ is the average density of air $\left(\mathrm{kg} / \mathrm{m}^{3}\right)$

$v_{t}$ : eddy viscosity $\left(\mathrm{m}^{2} / \mathrm{s}\right)$

$\hat{v}$ : the dimensionless eddy viscosity

$\hat{x} j=x j / L_{o}$ dimensionless coordinate in $\mathrm{j}$ component

$\hat{u} j=u j / U_{o}$ dimensionless velocity of air

$\hat{\rho}=\rho / \rho_{o}$ dimensionless density of air

$\hat{p}=p / \rho_{o} U_{o}^{2}$ dimensionless local pressure of air

$\hat{k}=k / U_{2}^{2}$ dimensionless turbulence energy

$\hat{\varepsilon}=\varepsilon L_{o} / U_{o}^{3}$ dimensionless dissipation rate of turbulent energy

$v_{t}=C_{D} \frac{\kappa^{2}}{\varepsilon}$ eddy viscosity

$\widehat{v}_{t}=\frac{v_{t}}{U_{o} L_{o}}$ dimensionless eddy viscosity

$v_{e f f}=\frac{1}{R_{e}}+\widehat{v}_{t}$ effective eddy viscosity

$\alpha_{e f f}=\frac{1}{R_{e} S_{c}}+\frac{\hat{v}_{t}}{\sigma_{c}}$ effective thermal diffusivity

$P_{k}=\frac{1}{R_{e}}+\frac{\hat{v}_{t}}{\sigma_{k}}$ effective turbulence energy diffusivity

$P_{\varepsilon}=\frac{1}{R_{e}}+\frac{\widehat{v}_{t}}{\sigma_{\varepsilon}}$ effective dissipation rate of turbulent energy diffusivity dimensionless

$\hat{V}_{g}=\frac{g d^{2} \rho_{p}}{18 \mu U_{0}}$ dimensionless gravitational settling velocity

$S_{c}=\frac{v}{D}$ Schmidt number

$\operatorname{Re}=L_{o} U_{o} / v$ Renold number

$p_{r}=\frac{v}{\alpha}$ Prantal number

Empirical constants: $\mathrm{c}_{\mathrm{D}}=0.09, \mathrm{c}_{1}=1.44, \mathrm{c}_{2}=1.92 \sigma_{\mathrm{x}}=$ $1.0, \sigma_{t}=1.3, \sigma_{c}=0.9$,

\section{REFERENCES}

1. Axley, J.W., "Adsorption Modeling for Building Contaminant Dispersal Analysis," Indoor Air, No. 2, pp. 147-171 (1991). 
2. Chen and Srebric, J., "Application of CFD Tools for Indoor and Outdoor Environment,', International Journal on Architectural Science, Vol. 1, No. 1, pp. 14-29 (2000).

3. Dunn, J.E., "Compensating for Sink Effects in Emissions Test Chambers by Mathematical Modeling," Atmospheric Environment, Vol. 22, No. 5, pp. 885-894 (1988).

4. Kurabuchi, T., Worker, G., Fang, J.B., and Grot, R.A., A Numerical Method for Calculating Indoor Airflows
Using a Turbulence Model, U.S Department of Commerce National Institute of standards and Technology, Gaithersburg, MD (1990).

5. Lin, L., "Numerical Analysis of Aerosol Removal for Different Convection Mechanisms in an Office Enclosure, " The Proceeding of 2001 Conference on Aerosol Science and Technology (2001).

6. Patankar, S.V., Numerical Heat Transfer and Fluid Flow, Hemisphere, Washington DC (1980). 